\title{
GENERATION AND PROPAGATION OF HIGH-ORDER BESSEL VORTICES IN LINEAR AND NON-LINEAR CRYSTALS
}

\author{
Vladimir N. Belyi ${ }^{1}$, Nikolai A. Khilo ${ }^{1}$, Andrew Forbes ${ }^{2,3}$, Anatol A. Ryzhevich ${ }^{1}$ \\ ${ }^{1}$ B.I. Stepanov Institute of Physics, NASB, 68, Nezavisimosti ave., 220072 Minsk, Belarus \\ ${ }^{2}$ CSIR National Laser Centre, PO Box 395, Pretoria 0001, South Africa \\ ${ }^{3}$ School of Physics, University of KwaZulu-Natal, Private Bag X54001, Durban 4000, South Africa
}

\begin{abstract}
Linear and nonlinear processes of transferring optical singularities from anisotropic crystals onto the wavefront of Bessel light beams (BLBs) are investigated. The generation of high-order vortices at the propagation of BLBs along the optical axes of crystals was studied. Particularly, a nonlinear frequency doubling of Bessel vortices in the conditions of new type synchronism (full conical phase-matching) is considered. This scheme of three-wave interactions of BLBs based on that the spatial frequency cones of BLBs coincide with phase-matching cones of uniaxial crystals. New type of frequency doubling of Bessel vortices has been experimentally realized in uniaxial crystals.
\end{abstract}

Key words: Bessel beam, optical vortex, second harmonic generation, phase matching, axicon, conical mirror.

\section{INTRODUCTION}

The most important BLBs property is the presence of screw dislocations of their wave front (Bessel vortices). Such dislocations are connected with the existence of orbital angular momentum of beam depending on the order of Bessel functions that describe electrical and magnetic BLB field [1-4]. The investigations of fields with orbital angular momentum are both of physical and applied interest. Particularly, in the latest years the phenomenon is studied of microparticles rotation by the mentioned beams and the possibilities are discussed of its application in optical tweezers [5-6].

Vortex Bessel beams are usually obtained using special holograms [7, 8]. There is also simple method of generation of vortex Bessel beams of the first and second orders, based on application of uniaxial [9] or biaxial [10] crystals. Here it is necessary that Bessel beams of the given polarization were propagated along optical axis of crystal. The peculiarity of beams obtained by such a method is rather low level of noises induced by the crystalline transformer. Consequently, crystal based generators of vortex beam can be interesting for observation of fine physical effects and also for some specific applications, for example, in metrology.

For obtaining BLB of higher orders with the use of crystals it is possible to apply the method of cascade increase of order due to repeated transmission of beam through the transformer. The other approach is based on the use of nonlinear (NL) processes, particularly, generation of harmonics and frequencies mixing.

But for realization of this approach, traditional geometry of frequency generation in crystals, which is applied for plane waves or Gaussian beams, can not be used here. In the present paper, axially symmetrical geometry of NL interactions in anisotropic crystals is investigated. This geometry is optimal as applied to Bessel beams having large cone angles.

\section{QUALITATIVE METHOD DESCRIPTION}

The peculiarity of using Bessel light beams for generation of the second harmonic consists, as is known, of necessity of fulfillment the conditions of transverse as well as longitudinal synchronisms (phase-matching) [11, 12]. In a case when BLB propagates along the direction of phase-matching for plane waves or Gaussian-type beams, the simultaneous fulfillment of these conditions, strictly speaking, is unrealizable. The reason for this is the presence of cone of wave vectors, angle at the vertex of which can be larger than angular width of phase-matching. The result of this is the

Laser Beam Shaping X, edited by Andrew Forbes, Todd E. Lizotte, Proc. of SPIE Vol. 7430 ,

$74300 \mathrm{~F} \cdot$ () 2009 SPIE · CCC code: 0277-786X/09/\$18 · doi: 10.1117/12.824851

Proc. of SPIE Vol. $743074300 \mathrm{~F}-1$ 
breaking of azimuthal symmetry of NL interaction and, as a consequence, intensity distribution of the second harmonic field. At that asymmetry strengthens when increasing cone angle.

There exists a simple way of achieving azimuthally symmetrical generation of the second harmonic (SHG) by Bessel beams. For this, it is necessary to go over to the geometry of interaction, where the direction of phase velocity of incident BLB coincides with the optical axis of crystal (see Fig.1). In addition, it is necessary to have the equality of BLB cone angle $\gamma$ and cone angle $\theta_{s}$ of phase-matching direction in uniaxial crystal.

In the scheme shown in Fig.1 the process of oo-e type SHG takes place due to collinear interaction, which applied

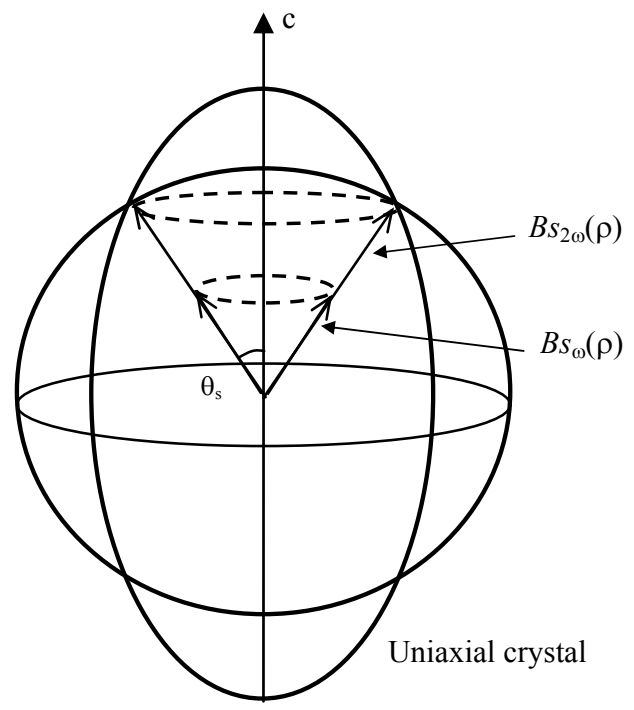

Figure 1: Geometry of SHG when wave vectors cone of Bessel beam at fundamental $B S_{\omega}(\rho)$ and second harmonic frequencies $B s_{2 \omega}(\rho)$ coincides with phase-matching cone of uniaxial crystal.

to BLB has, as is known, high efficiency [13]. The generation of harmonic is fulfilled in conditions of longitudinal synchronism simultaneously along all azimuths within the region of $(0 \div 2 \pi)$. Due to axial symmetry of interaction the second harmonic field is also Bessel beam with the same cone angle $\theta_{\mathrm{s}}$ that provides maximum of overlap integral with BLB of fundamental frequency [11]. Note that the analysis of oe-e type interaction is fully analogical.

\section{THEORETICAL ANALYSIS OF A SCHEME. CRYSTALS OF HEXAGONAL SYMMETRY}

As Fig. 1 shows the peculiarity of full azimuth SHG is the necessity of using BLB with rather large cone angle. For example, for crystal of BBO, the angle is equal to approximately $23 \mathrm{deg}$ for radiation with $\lambda=1.06 \mathrm{mkm}$. That is why for correct calculation of the SGH process it is necessary to perform full vectorial analysis.

Optimal conditions are realized for generation of high order Bessel vortices at SHG, when circularly polarized Bessel beam incidences on crystal. From the Maxwell equations it follows, that transverse component of electrical field for such beams can be found in form $\mathbf{E}_{i \perp}(\rho, \varphi, z)=\mathbf{e}_{+} J_{0}(q \rho) \exp \left(i k_{z} z\right)$. Here and further the following designations are used: $J_{m}(q \rho)$ is m-order Bessel function of first type, $k_{z}=k_{0} n \cos (\gamma),(\rho, \varphi, z)$ are cylindrical coordinates, $\mathbf{e}_{ \pm}$are unit vectors of right and left circular polarization.

From boundary conditions it follows that the incident beam excites in the crystal of TE- and TH-polarized BLBs (further are marked by indices "o" and "e", correspondingly) with the same power on its entrance. The transverse components $E_{\perp 0}$ and $E_{\perp e}$ of the electric field for these beams are given by the following expressions:

$$
\mathbf{E}_{\perp o}=i \frac{A_{10} \exp \left(i k_{o z} z\right)}{\sqrt{w_{1}(q)}}\left(J_{0}(q \rho) \mathbf{e}_{+}+J_{2}(q \rho) \mathbf{e}_{-} \exp (2 i \varphi)\right)
$$


$\mathbf{E}_{\perp e}=i \frac{A_{1 e} \exp \left(i k_{e z} z\right)}{\sqrt{w_{1}(q)}} \cos \left(\gamma_{e}\right)\left(J_{0}(q \rho) \mathbf{e}_{+}-J_{2}(q \rho) \mathbf{e}_{-} \exp (2 i \varphi)\right)$

where $A_{10}$ and $A_{l e}$ are amplitudes,

$w_{1}(q)=2 \pi \int_{0}^{R}\left(J_{0}^{2}(q \rho)+J_{2}^{2}(q \rho)\right) \rho d \rho$

is normalization multiplier, $R$ is beam radius. Here longitudinal components $E_{o, z}=0$, $E_{e z}=2\left(\varepsilon_{o} / \varepsilon_{e}\right) \sin \left(\gamma_{e}\right) J_{1}(q \rho) \exp [i \varphi]$, where $\varepsilon_{\mathrm{o}}$ and $\varepsilon_{\mathrm{e}}$ are principal values of dielectric permittivity tensor of the crystal, $k_{o z}=k_{0} n_{o} \cos \left(\gamma_{o}\right), k_{e z}=k_{0} n_{e} \cos \left(\gamma_{e}\right), q=k_{o} n_{o, e} \sin \left(\gamma_{o, e}\right), n_{o}^{2}=\varepsilon_{o}, n_{e}^{2}(\gamma)=\varepsilon_{o} \varepsilon_{e} /\left(\varepsilon_{o} \sin ^{2}(\gamma)+\varepsilon_{e} \cos ^{2}(\gamma)\right)$.

It should be noted that TH- and TE-waves in crystal contain the component with azimuthal dependence $\exp (2 i \varphi)$, while in incident beam it is absent.

Using Eq. (1) there has been made the calculation of vector of non-linear polarization $\mathbf{P}_{2}$ at the double frequency for oo-e type of synchronism for hexagonal symmetry crystals of $\mathrm{C}_{6}$ point group

$\mathbf{P}_{2}(\rho, \varphi, z)=A_{10}(z) A_{1 e}(z) \mathbf{f}_{2}(\rho, \varphi) \exp \left[i\left(k_{0 z}+k_{e z}\right) z\right]$,

where $\mathbf{f}_{2}(\rho, \varphi)=\frac{2 d \sin \left(\gamma_{e}\right)}{w_{1}\left(q_{1}\right)} \frac{\varepsilon_{o}(\omega)}{\varepsilon_{e}(\omega)} J_{1}(q \rho)\left(\mathbf{e}_{+} J_{0}\left(q_{1} \rho\right) \exp \left[i\left(\varphi-\varphi_{0}\right)\right]-\mathbf{e} J_{2}\left(q_{1} \rho\right) \exp \left(3 i \varphi+i \varphi_{0}\right)\right)$,

$d=\sqrt{d_{14}^{2}+d_{15}^{2}}, \operatorname{tg}\left(\varphi_{0}\right)=d_{15} / d_{14}$.

As is seen from Eq. (2), going over to vector BLBs additionally involves in the SHG process the component of nonlinear dielectric susceptibility $d_{15}$. Its appearance is determined by nonzero azimuthal component of electric field of TH Bessel beam. Note that this component is absent in extraordinary plane wave. This peculiarity of vector BLB opens, principally, the perspective of increasing the efficiency of three-wave interaction due to use of maximal values of NL coefficients.

Based on the form of NL polarization (2), spatial structure can be specified of generated TH-beam of second harmonic. In common case the solution of Maxwell equation for e-wave in crystal can be written as

$\mathbf{E}_{e m}=i A_{e} \cos \left(\gamma_{e}\right)\left(J_{m-1}(q \rho) \exp [i(m-1) \varphi] \mathbf{e}_{+}-J_{m+1}(q \rho) \exp [i(m+1) \varphi] \mathbf{e}_{-}\right)$.

The comparison of Eqs. (2) and (3) gives that the coincidence of their azimuthal dependence takes place at $m=2$. Consequently, the second harmonic field can be represented as

$\mathbf{E}_{\mathbf{2}}(\rho, \varphi, z)=i \frac{A_{2}(z)}{\sqrt{w_{2}\left(q_{2}\right)}} \cos \left(\gamma_{e}\right)\left(J_{1}\left(q_{2} \rho\right) \exp (i \varphi) \mathbf{e}_{+}-J_{3}\left(q_{2} \rho\right) \mathbf{e}_{-} \exp (3 i \varphi)\right) \exp \left(i k_{2 z} z\right)$,

where $w_{2}\left(q_{2}\right)=2 \pi \int_{0}^{R}\left(J_{1}^{2}\left(q_{2} \rho\right)+J_{3}^{2}\left(q_{2} \rho\right)\right) \rho d \rho$.

Here the function $A_{2}(z)$ is described by the following equation for slowly varying amplitudes

$\frac{\partial A_{2}(z)}{\partial z}=i A_{1 o} A_{1 e}\left(g_{011}\left(q_{2}\right)+g_{123}\left(q_{2}\right)\right) \exp \left[-i \Delta k_{z} z\right]$

where $\Delta k_{z} z=k_{2 z}-k_{o z}-k_{e z}$. 
The peculiarity of the SHG process with the participation of vector Bessel beams is relatively complex structure of overlap integrals that determine so called transverse phase-matching of interaction beams. In the examined case overlap integrals are described by the following expressions:

$$
\begin{aligned}
& g_{011}\left(q_{2}\right)=\frac{2 d \varepsilon_{o}(\omega) \operatorname{tg}\left(\gamma_{e}\right) \exp \left(-i \varphi_{0}\right)}{w_{1}\left(q_{1}\right) \sqrt{w_{2}\left(q_{2}\right)} \varepsilon_{e}(\omega)} \int_{0}^{R} J_{0}\left(q_{1} \rho\right) J_{1}\left(q_{1} \rho\right) J_{1}\left(q_{2} \rho\right) \rho d \rho \\
& g_{123}\left(q_{2}\right)=\frac{2 d \varepsilon_{o}(\omega) \operatorname{tg}\left(\gamma_{e}\right) \exp \left(i \varphi_{0}\right)}{w_{1}\left(q_{1}\right) \sqrt{w_{2}\left(q_{2}\right)} \varepsilon_{e}(\omega)} \int_{0}^{R} J_{0}\left(q_{1} \rho\right) J_{2}\left(q_{1} \rho\right) J_{3}\left(q_{2} \rho\right) \rho d \rho .
\end{aligned}
$$

As is seen from the subintegral expressions in (6), the overlap integrals $g_{011}\left(q_{2}\right)$ and $g_{123}\left(q_{2}\right)$ are responsible for generation of $J_{1}$ and $J_{3}$ component of a beam of double frequency correspondingly by $J_{0}$ and $J_{1}$ components, and also $J_{1}$ and $J_{2}$ of the field at the fundamental frequency. Numerical simulation of integrals (6) shows that they have the typical maximum at $q_{1}+q_{2}=q_{3}$, which value coincides approximately with the corresponding value for interaction of beams of zeroth-order [14]. Note that the above relation between transverse wave numbers $q_{1}+q_{2}=q_{3}$ indicates to collinear type of synchronism for plane-wave component of Bessel beams (see also Fig.1).

From the comparison of the field incident on NL crystal and the second harmonic field it follows that in this NL process there occurs the transformation of Bessel function order (see Fig.2).

Thus, in crystals of the $\mathrm{C}_{6}$ class symmetry at oe-e interaction there takes place the following transformation of order of Bessel beam (or generation of high-order Bessel vortices)

$J_{0}(\omega) \mathbf{e}_{+} \rightarrow J_{1}(2 \omega) \exp (i \varphi) \mathbf{e}_{+}+J_{3}(2 \omega) \exp (3 i \varphi) \mathbf{e}_{-}$

Let us further consider the interaction of oo-e type. The calculation of NL polarization at the double frequency gives $p_{2 \mathrm{x}}=0, p_{2 \mathrm{y}}=0$. Longitudinal component is non-zero and equals to

$p_{2 z}=-4 d_{15} A_{1}^{2} J_{0}\left(q_{1} \rho\right) J_{2}\left(q_{1} \rho\right) \exp [2 i \varphi] \exp \left[2 i k_{o z} z\right]$.

It is necessary to point out that this component of NL polarization is absent in a case of plane waves, and its appearance is determined by the difference of polarization of ordinary and extraordinary plane waves and TE- TH-

$$
J_{0}(\omega) \mathbf{e}_{+} \quad J_{1}(2 \omega) \mathbf{e}_{+} \quad J_{3}(2 \omega) \mathbf{e}_{-}
$$

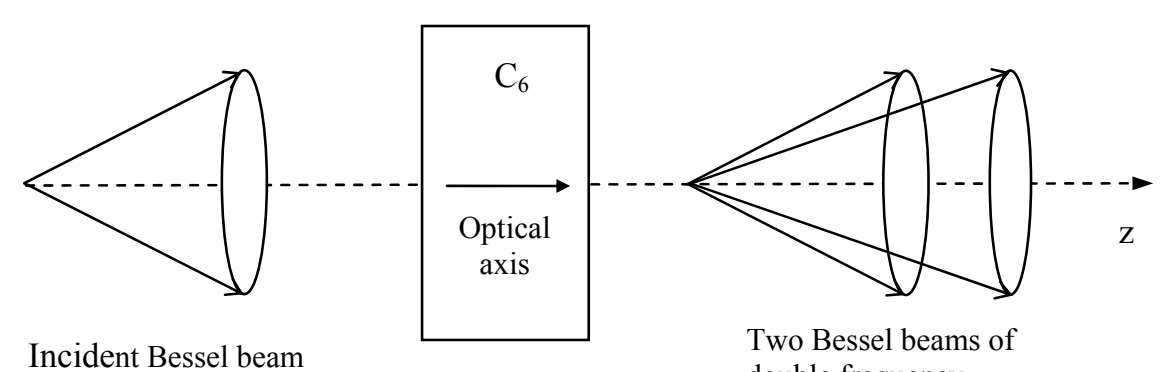

Incident Bessel beam double frequency

Figure 2. Transformation of the order of Bessel function at oe-e interaction of vector Bessel beams in uniaxial crystal of $C_{6}$-symmetry.

polarized BLBs. From the known NL polarization one can find the longitudinal component of generated field of second harmonic $E_{2 z} \sim J_{2}\left(q_{2} \rho\right) \exp (2 i \varphi)$. The transverse component is obtained from the solution of the Maxwell equations and 
coincides with Eq. (4). Consequently, in the examined process of frequency doubling there occurs the transformation of the Bessel function order shown in Fig.2.

\section{SHG IN TRIGONAL CRYSTALS}

The non-linear polarization vector on double-frequency for o-ee synchronism in crystals of symmetry $3 \mathrm{~m}$ point group can be represented in the following form

$\mathbf{P}_{2}(\rho, \varphi, z)=A_{1 o}(z) A_{1 e}(z) \mathbf{f}_{2}(\rho, \varphi) \exp \left[i\left(k_{o z}+k_{e z}\right) z\right]$,

where

$\mathbf{f}_{2}(\rho, \varphi)=\frac{2 i}{w_{1}\left(q_{1}\right)}\left\{\begin{array}{l}d_{15} \sin \left(\gamma_{e}\right) \frac{\varepsilon_{o}(\omega)}{\varepsilon_{e}(\omega)} J_{1}(q \rho)\left[\mathbf{e}_{+} J_{0}\left(q_{1} \rho\right) \exp [i \varphi]+\mathbf{e}_{-} J_{2}\left(q_{1} \rho\right) \exp [i 3 \varphi]\right]+ \\ +d_{22} \cos \left(\gamma_{e}\right)\left[\mathbf{e}_{-} J_{0}^{2}\left(q_{1} \rho\right)+\mathbf{e}_{+} J_{2}^{2}\left(q_{1} \rho\right) \exp [4 i \varphi]\right.\end{array}\right\}$.

From (7) it is found that the second harmonic field is the superposition of two component

$\mathbf{E}_{2}(\rho, \varphi)=\mathbf{E}_{2}^{-}(\rho, \varphi)+\mathbf{E}_{2}^{+}(\rho, \varphi)$,

where

$\mathbf{E}_{2}^{-}(\rho, \varphi)=-\left(J_{0}\left(q_{2} \rho\right)+J_{3}\left(q_{2} \rho\right) \exp (3 i \varphi)\right) \mathbf{e}_{-}$,

$\mathbf{E}_{2}^{+}(\rho, \varphi)=\left(J_{1}\left(q_{2} \rho\right) \exp (i \varphi)+J_{4}\left(q_{2} \rho\right) \exp (4 i \varphi)\right) \mathbf{e}_{+}$.

As is seen there are two channels (Fig. 3) in second harmonic radiation: one contains left-circularly polarized

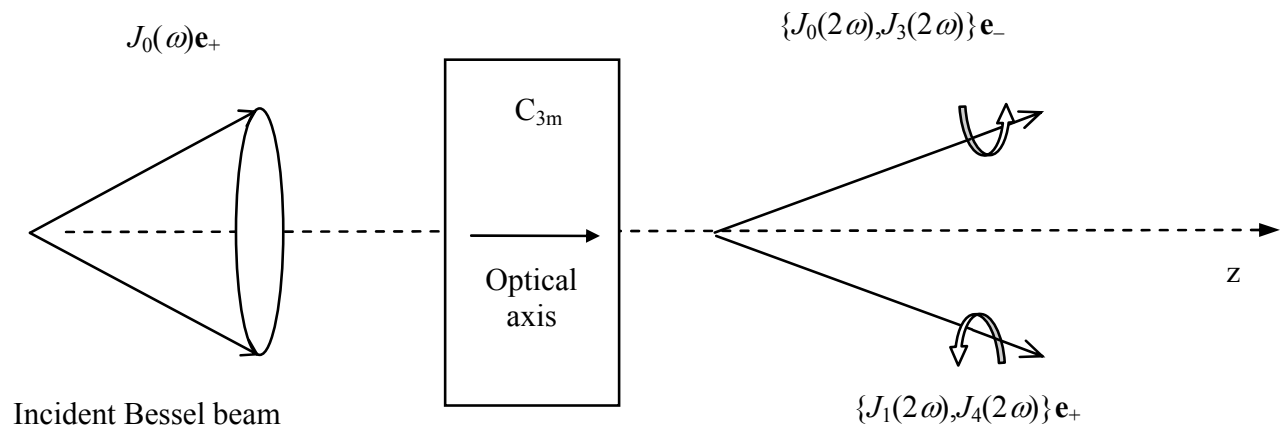

Figure 3. Superposition of 4 BLBs of different orders generated by circularly polarized BLB of zeroth order

SH field, the other contains right-circularly-polarized SH field. Using Eqs. (7)-(8) it is feasible to obtain equations for slowly varying amplitudes in a usual way and to calculate the overlap integrals. But yet from the kind of solution of Eq. (8) it follows that oe-e type of interaction in crystals $3 \mathrm{~m}$ is accompanied by rich set of transformations of Bessel function order. Namely, right-circular polarized zeroth-order BLB generates the superposition of Bessel vortices of first and 
fourth orders with polarization orthogonal to the incident ones. Moreover, additional third order Bessel vortex generates with the polarization coincident of incident beam (see Fig.3).

The investigation of amplitude-phase structure of the generated BLB superposition is of great interest. In accordance with Eqs. (9) and (10) the second harmonic intensity is azimuthally inhomogeneous and governs by the equation

$I(x, \varphi) \sim J_{0}^{2}(x)+J_{1}^{2}(x)+J_{3}^{2}(x)+J_{4}^{2}(x)+2\left(J_{0}(x) J_{3}(x)+J_{1}(x) J_{4}(x)\right) \cos (3 \varphi)$,

where $x=q_{2} \rho$.

Interference pictures produced by second harmonic radiation and spherical reference wave are shown in Figs. 4, 5.

As is seen in the field depicted on Fig. 4a, vortex is localized in narrow central area. In a case of interference with BLB of third order (Fig. 4c), with BLB of first order (Fig. 5a) and the fourth order (Fig. 5b) it is observed helical structure with additional modulation. Here the axial vortex is shifted from the beam. In the vicinity of axis there appears $m$ vortex, where $m$ is the order of BLB. The occurrence of BLB of zeroth order with the maximum in the center in Eq. (10) suppresses local minimum of intensity in the beam center (Fig. 4d). Mutual influence of vortices of first and fourth order in Eq. (9) (Fig. 5c) destroys the helical picture (Fig. 5b). The resulting intensity of field of second harmonic is characterized by azimuthal modulation of third order and absence of intensity nulls.

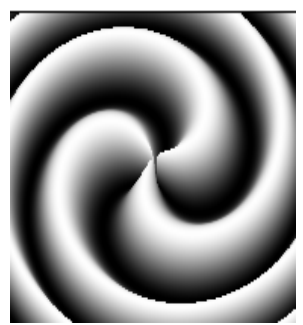

a)

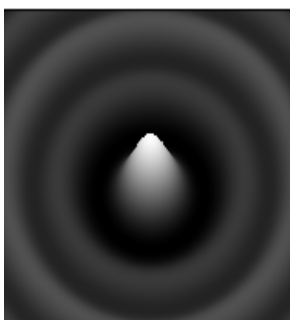

b)

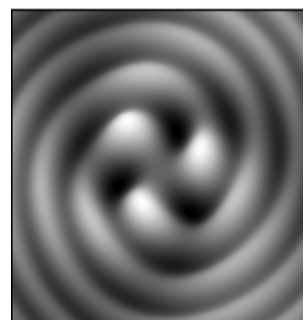

c)

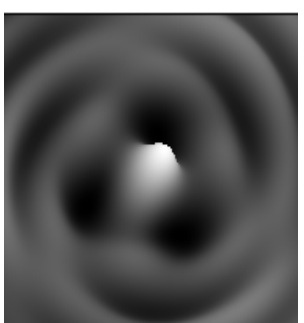

d)

Figure 4. Spatial structure of the left-polarized component of the second-harmonic vortex field revealed at interference with spherical reference wave. a) - interference with the field $\exp (3 i \varphi), b)$ - with zeroth-order BLB in (9), c) - with the third-order BLB in (9), d) - with total field (9).

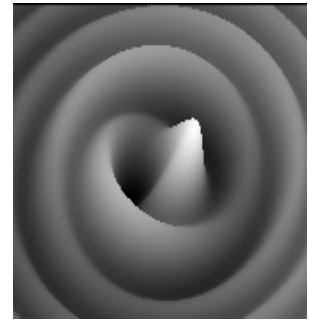

a)

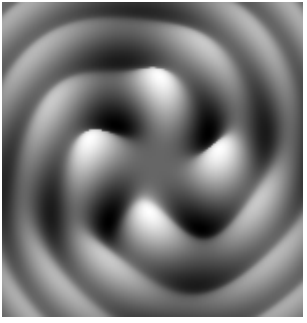

b)

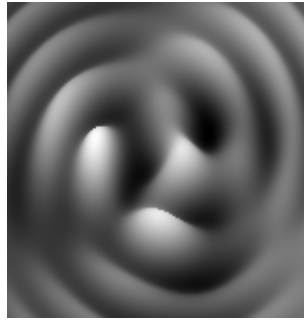

c)

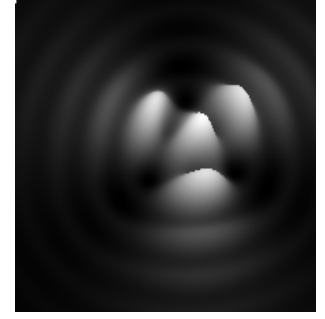

d)

Figure 5. Spatial structure of the right-polarized component of the second-harmonic vortex field revealed at interference with spherical reference wave. a) - interference with the first-order BLB, b) - with fourth-order BLB in (10), c) - with the total field in (10), d) - spatial distribution of the intensity of the second harmonic field (8).

\section{EXPERIMENT}

For experimental realization of full azimuthal synchronism the BBO crystal was chosen. This is uniaxial crystal of $3 \mathrm{~m}$ point group symmetry and phase-matching angle of $\theta_{\mathrm{s}}=22.8 \mathrm{deg}$ for $\lambda=1064 \mathrm{~nm}$. The crystal was cut perpendicular to the optical axis and has the thickness of $5 \mathrm{~mm}$. Angular width of synchronism $\delta \theta_{\mathrm{s}}$ was equal to $0.51 \mathrm{mrad}$ that practically excludes the possibility of SHG realization in traditional scheme, when Bessel beam propagates along the synchronism direction. 
For formation of BLB at fundamental frequency with necessary cone angle in air $\quad=39.9^{\circ}$ there was used

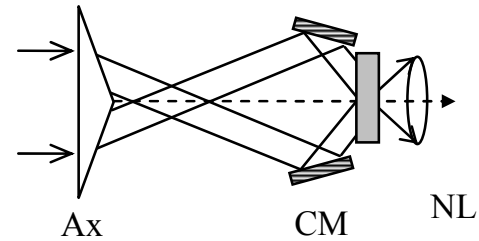

a)

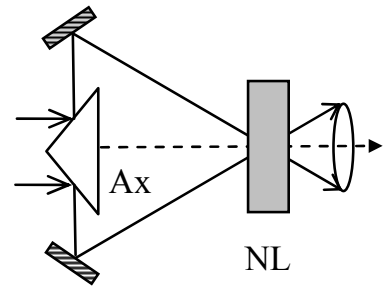

$\mathrm{CM}$

b)

Figure 6. Variants of experimental scheme: Ax - axicon (refractive (a) and reflective (b)), CM - conical mirror, NLC - nonlinear crystal (BBO).

refractive axicon with the base angle of $5 \mathrm{deg}$ and specially manufactured conical mirror for increasing cone angle up to necessary value (Fig. 6a). It should be noted that for obtaining BLB with large cone angles it is perspective the application of reflective axicons in combination with conical mirror (Fig. 6 b).

Radiation from a $1.064 \mu \mathrm{m} \mathrm{Nd}$ : YAG laser is a Gaussian beam with angular divergence of $\theta \approx 0.8 \mathrm{mrad}$, pulse duration of $50 \mathrm{~ns}$, and energy of $20 \mathrm{~mJ}$.

Fig. $7 \mathrm{~b}$ shows the experimentally registered intensity distribution of the second harmonic in far zone of radiation obtained with the application of optical Fourier transformation. For comparison, Fig. 7a shows the Fourier-spectrum field $E(\rho, \varphi)$ described by Eq. (8). The key feature of the order of generated Bessel function is seen to be the number of lobes in the intensity distribution that proves the calculation made before.
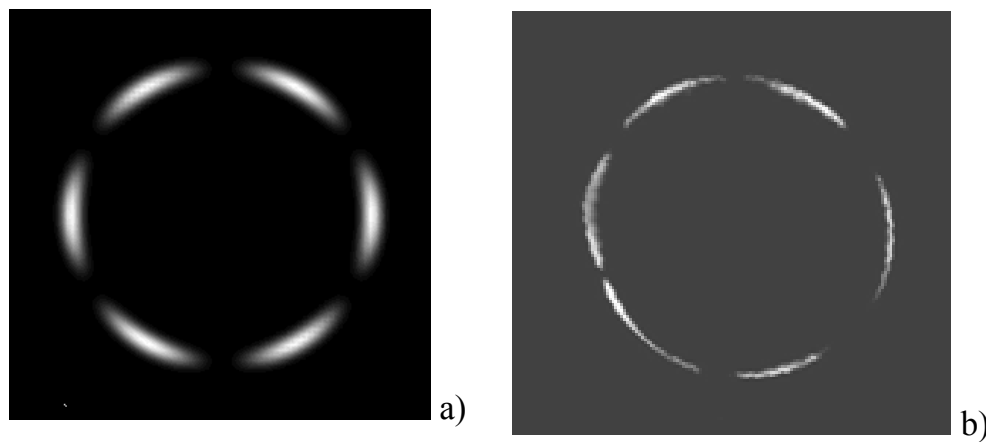

Figure 7. Far-field intensity distribution of second harmonic generated in BBO crystal: a) calculation, b) - experiment.

\section{CONCLUSIONS}

Nonlinear processes of transferring optical singularities from anisotropic crystals onto the wavefront of Bessel light beams (BLB) are investigated, with particular reference to the generation of high-order vortices in Bessel beams.

It has been considered frequency doubling of Bessel vortices in the new condition of full conical phase matching. This scheme of three-wave interactions of quasi-nondiffractive Bessel light beams is based on the property that the spatial frequency cones of Bessel beams may be made to coincide with the phase-matching cones of uniaxial crystals. Also this scheme allows putting into practice nonlinear frequency transformation of Bessel beams having a cone angle of several tens of degrees. Peculiarities of frequency doubling of Bessel vortices in the conditions of full conical phase-matching have been investigated for uniaxial crystals of hexagonal and trigonal symmetry. This new type of frequency doubling of Bessel vortices has been experimentally realized in uniaxial crystal BBO when the incident zeroorder Bessel light beams at fundamental frequency is directed along the optic axis of crystal and its cone angle is equal to the phase-matching angle.

The investigated in the paper SHG process by Bessel beams in conditions of full azimuthal synchronism allows one to generate Bessel beams of various orders (Bessel vortices) and their linear superpositions. The transformation of 
Bessel beam order at frequency doubling is the result of taking into account all plane wave components of vector BLBs, which, in common case, are described by different Bessel functions. Here the selection of field structure of second harmonic is realized by means of mechanism of transverse synchronism, while longitudinal synchronism in the scheme under study does not depend on azimuthal angle. The examined in the paper particular cases of hexagonal and trigonal symmetry crystals $\left(\mathrm{C}_{6}\right.$ and $3 \mathrm{~m}$ point group) differ by axial symmetry of the effective nonlinear dielectric susceptibility ( $d_{\text {eff }}$ - coefficient). In common case there is azimuthal dependence of SHG efficiency, when $d_{\text {eff }}=d_{\text {eff }}(\varphi)$. The result of this would be additional azimuthal modulation of the second harmonic field and novel possibilities of transformation of Bessel vortices.

The advantage of axial-symmetrical scheme is the absence of walk-off effect and caused by them distortion of second harmonic intensity distribution. Consequently, such crystal-based transformers of Bessel beam order, like linear analogues, would be characterized by high quality of output optical signal.

It is necessary to notice that the generated vector Bessel beams are characterized by rather small-scale ring structure. Thus in the described before experiment the diameter of main maximum of BLB of zeroth order is equal to $0.58 \mathrm{mkm}$, and the diameter of dark axial field for BLB of first order is equal to $0.93 \mathrm{mkm}$. Such beams can be interesting for application in microscopy and for creation of optical tweezers.

\section{REFERENCES}

[1] Z. Bouchal and M. Olivık, "Non-diffractive vector Bessel beams," J. Mod. Opt. Vol. 42, 1555-1566 (1995).

[2] J. Turunen and A. T. Friberg, "Self-imaging and propagation-invariance in electromagnetic fields," Pure Appl. Opt. Vol. 2, 51-60 (1993).

[3] S. R. Mishra, “A vector wave analysis of a Bessel beam,” Opt. Communs. Vol. 85, 159-161 (1991).

[4] Z. Bouchal, R. Horak and J. Wagner, "Propagation-invariant electromagnetic fields," J. Mod. Opt. Vol. 43, 19051920 (1996).

[5] D. McGloin, V. Garces-Chavez, K. Dholakia, "Interfering Bessel beams for optical micromanipulation," Opt. Lett. Vol. 28, 657-659 (2003).

[6] S.N. Khonina, V.V. Kotlyar, R.V. Skidanov, K. Jefimovs, J. Simonen, J. Turunen, "Ratation of microparticles with Bessel beams generated by diffractive elements," J. Mod. Opt. Vol. 51, 2167-2184 (2004).

[7] A. Vasara, J. Turunen, A.T. Friberg, "Realization of general nondiffracting beams with computer-generating holograms,” J. Opt. Soc. Am. Vol. A 6, 1748-1754 (1989).

[8] J. Tervo and J. Turunen, "Generation of vectorial propagation-invariant fields by polarization grating axicons," Opt. Communs. 192, 13-18 (2001).

[9] N.A Khilo, A.A. Ryzhevich, E.S. Petrova, "Transformation of the order of Bessel light beams in uniaxial crystals," Quantum electronics. Vol. 31(1), 85-89 (2001).

[10] N S Kazak, N A Khilo, A A Ryzhevich, "Generation of Bessel light beams under the conditions of internal conical refraction," Quantum Electronics Vol. 29 (11), 1020 - 1024 (1999).

[11] V N Belyi, N S Kasak, N A Khilo, "Frequency conversion of Bessel light beams in nonlinear crystals," Quantum Electronics Vol. 30(9), 753 - 766 (2000).

[12] R. Gadonas, V. Jarutis, A. Marcinkevicius, V. Smilgevichius, A. Stabini, “Angular distribution of second harmonic generated by Bessel beam," Opt. Communs. Vol. 167, 299-309 (1999).

[13] V N Belyi, N S Kazak, N V Kondratyuk, N A Khilo, A A Shagov, "Generation of the second harmonics of Bessel light beams in a KTP crystal," Quantum Electronics, Vol. 28, 1011-1016 (1998). 